Supporting Information for

\title{
Core-Tetrasubstituted Naphthalene Diimides: Synthesis, Optical Properties, and Redox Characteristics
}

Cornelia Röger and Frank Würthner*

Universität Würzburg, Institut für Organische Chemie, Am Hubland, 97074 Würzburg, Germany

E-mail: wuerthner@chemie.uni-wuerzburg.de

\section{Table of Contents}

Materials and Methods

General

Absorption and Fluorescence Spectroscopy

Electrochemistry

Additional References

Measured and calculated EI mass spectra of $\mathbf{4}$

${ }^{1} \mathrm{H}$ NMR spectrum of NDI 5

${ }^{1} \mathrm{H}$ NMR spectrum of NDI 6

${ }^{1} \mathrm{H}$ NMR spectrum of NDI 7

${ }^{1} \mathrm{H}$ NMR spectrum of NDI 8

${ }^{1} \mathrm{H}$ NMR spectrum of NDI 9 


\title{
Materials and Methods
}

General. Solvents and reagents were obtained from commercial sources, unless otherwise stated, and purified and dried according to standard procedures. ${ }^{\mathrm{S} 1}$ Dibromoisocyanuric acid (DBI) was synthesized according to literature. ${ }^{\text {S2 }}$ Column chromatography was performed on silica gel (Silica Gel 60, mesh size $0.2-0.5 \mathrm{~mm}$ ) and high performance liquid chromatography (HPLC) was performed on a reversed phase column (RP18). NMR spectra were recorded on a $400 \mathrm{MHz}$ spectrometer using the residual solvent peak as internal standard.

\begin{abstract}
Absorption and Fluorescence Spectroscopy. UV-vis and fluorescence spectra were taken on commercial spectrometers. All fluorescence spectra are corrected. The solvents for spectroscopic studies were of spectroscopic grade and used as received. The fluorescence quantum yields in $\mathrm{CH}_{2} \mathrm{Cl}_{2}$ were determined by the optically dilute method ${ }^{\mathrm{S} 3}$ using either cresyl violet perchlorate $\left(\Phi_{f}(\mathrm{MeOH})=0.54\right),{ }^{\mathrm{S} 4}$ or 9,10 -diphenylanthracene $\left(\Phi_{f}\right.$ (cyclohexane) $=0.95)^{\mathrm{S} 5}$ as a reference and refractive indexes of the solvents as published in the CRC handbook. ${ }^{\mathrm{S} 6}$ The given quantum yields were averaged from values obtained at three excitation wavelengths with a standard deviation $\sigma$ of less than $3 \%$.
\end{abstract}

Electrochemistry. Cyclic voltammetry was performed with a standard commercial electrochemical analyser in a three electrode single-compartment cell under argon. Working electrode: platinum disk; counter electrode: platinum wire; reference electrode: $\mathrm{Ag} / \mathrm{AgCl}$. All potentials were internally referenced to the $\mathrm{Fc} / \mathrm{Fc}^{+}$couple. Dichloromethane (HPLC grade) was used as solvent that was dried over calciumhydride and degassed prior to measurement. The supporting electrolyte was $0.1 \mathrm{M}$ tetrabutylammonium hexafluorophosphate, which was recrystallized twice from ethanol / water and dried in a high vacuum. 


\section{Additional References}

(S1) Perrin, D. D.; Armarego, W. L. F. Purification of Laboratory Chemicals, $2^{\text {nd }}$ ed.; Pergamon Press: Oxford, 1980.

(S2) Becker, H. G. O. et al. Organikum, 19 ${ }^{\text {th }}$ Ed.; Barth: Leipzig, 1993; pp. 329-333.

(S3) Lakowicz, J. R. Principles of Fluorescence Spectroscopy, $2^{\text {nd }}$ ed.; Kluwer Academic/ Plenum Publishers: New York, 1999; pp. 52-53.

(S4) Magde, D.; Brannon, J. H.; Cremers, T. L.; Olmsted III, J. J. Phys. Chem. 1979, 83, 696-699.

(S5) Mardelli, M.; Olmsted III, J. J. Photochem. 1977, 7, 277-285.

(S6) Lide, D. R. CRC Handbook of Chemistry and Physics, $72^{\text {nd }}$ ed.; CRC Press: Boca Raton, 1991. 


\section{EI-MS spectrum of compound 4}

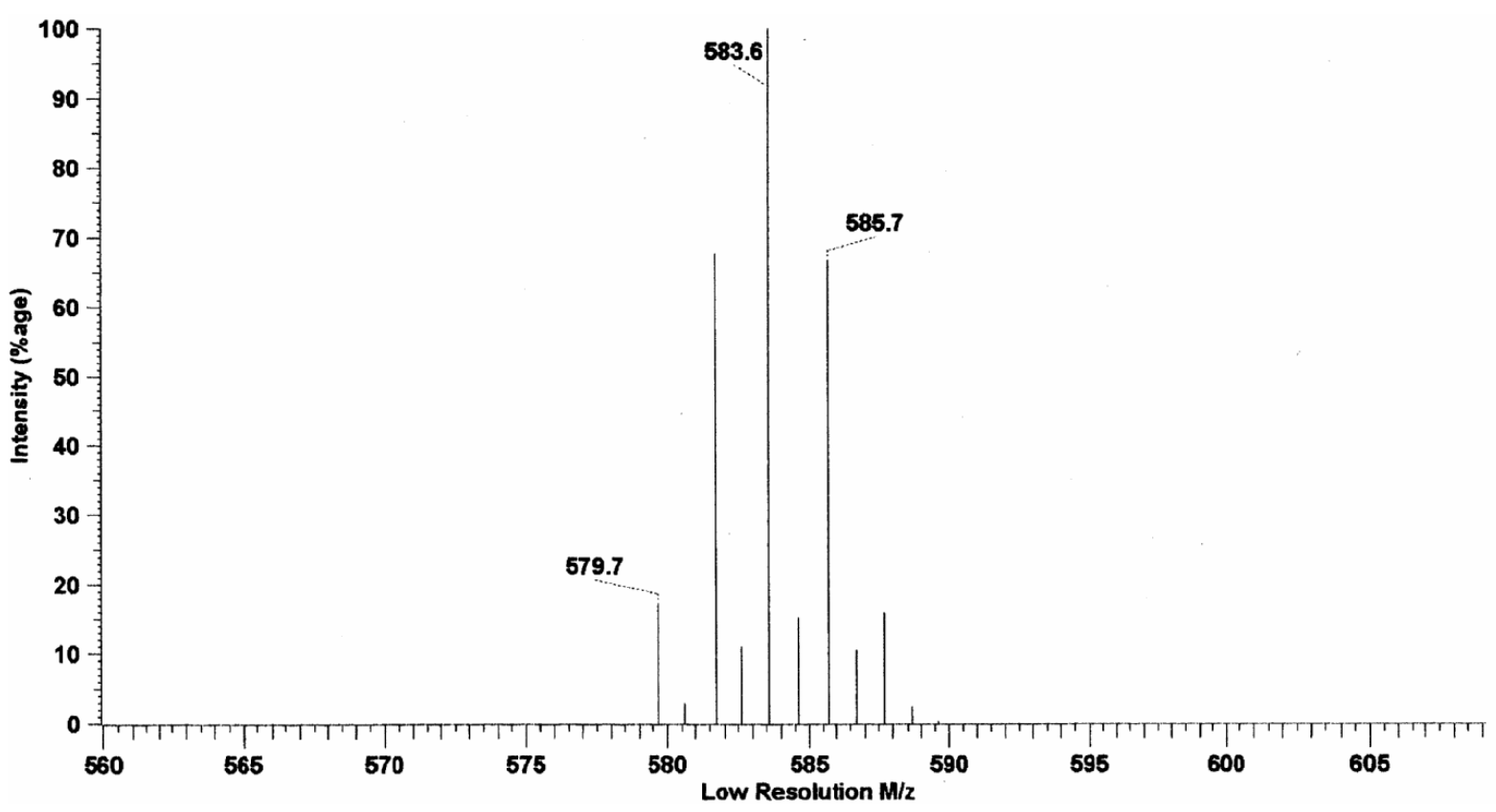

Figure S1. Isotope pattern of the $\mathrm{M}^{+}$signal of compound 4 measured by El mass spectrometry.

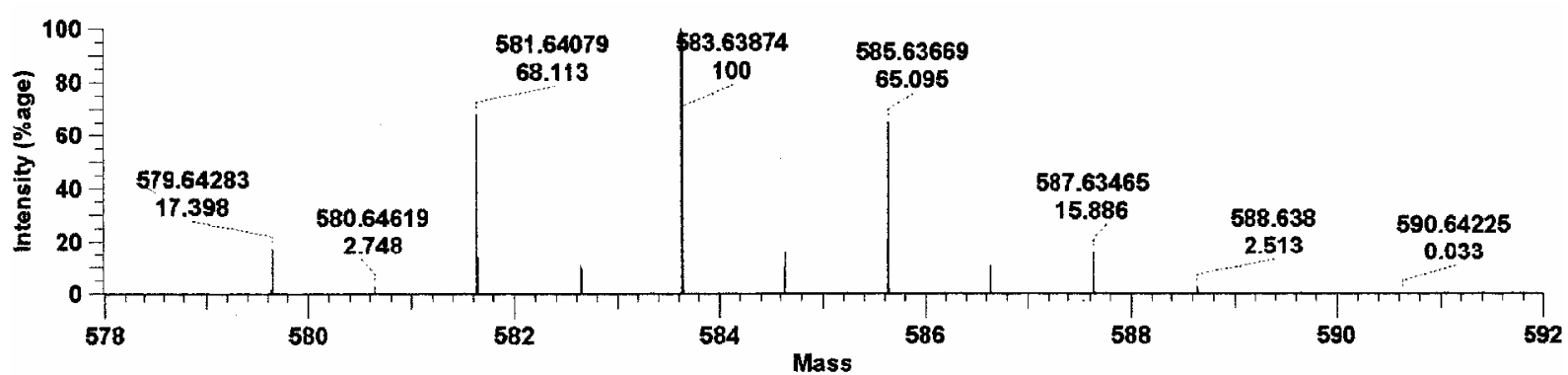

Figure S2. For comparison, the calculated isotope pattern of the $\mathrm{M}^{+}$signal of $\mathbf{4}$ is depicted. 
NDI 5

$\begin{array}{lllll}t & S & 6 & I & \cdot \\ 8 & \tau & I & Z & I\end{array}$

$\begin{array}{lllll}I & z & \varepsilon & 9 & 7 \\ 8 & 8 & + & 9 & 7 \\ z & 9 & 9 & 9\end{array}$

$z 999 \cdot z$

$z \mathcal{E} 89^{\circ} z$

$\varepsilon 00 \mathrm{~L} \cdot \mathrm{Z}$

$\left.\begin{array}{llll}\mathcal{E} L & I & \cdot & \cdot \\ t & \dagger & \mathcal{E} & L\end{array}\right]$
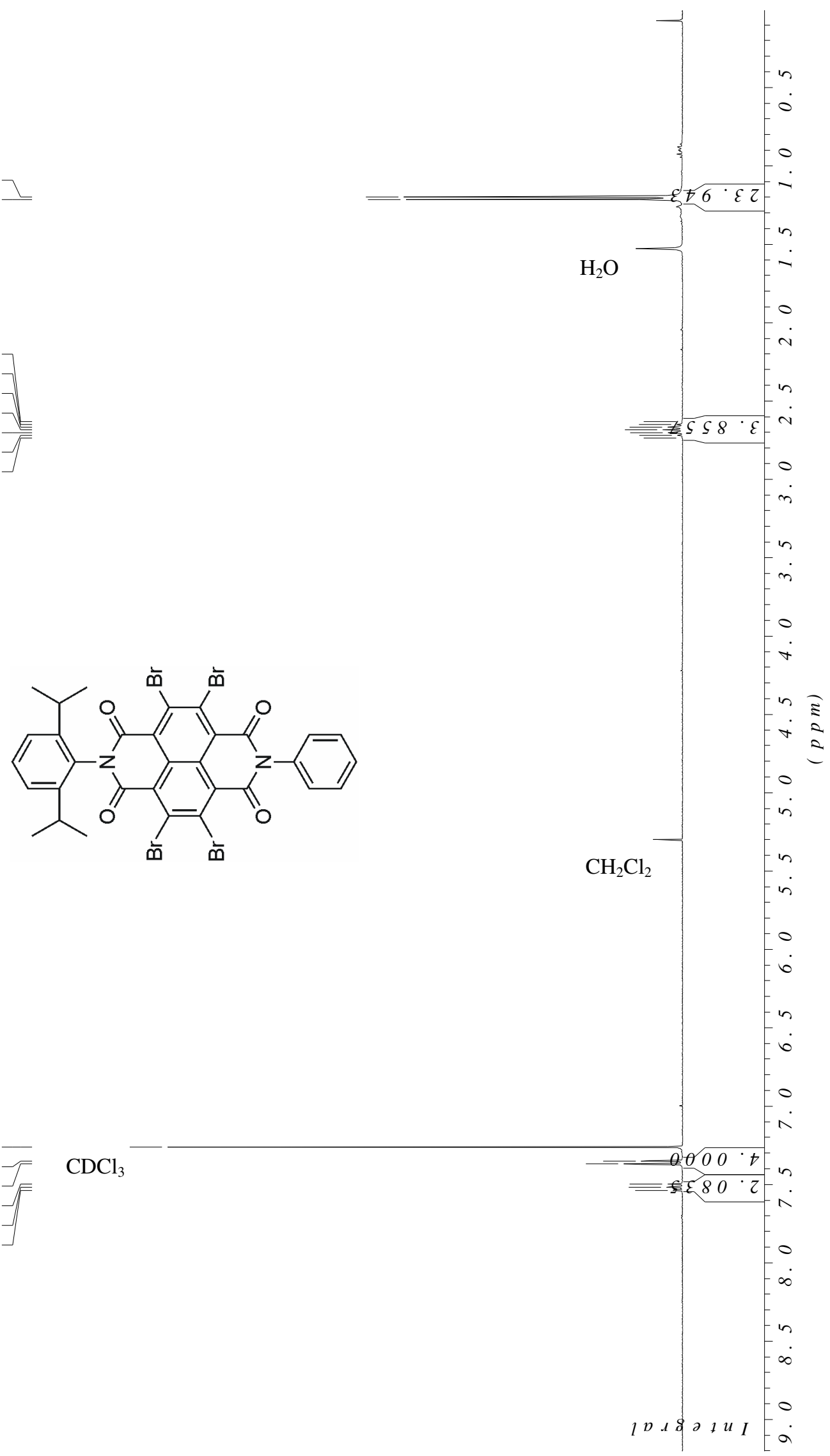

$0092 \cdot L$ $\hbar 8+\mathcal{E} \cdot L-$

t96t. $\mathrm{L}$

09 IS. $L$

$\mathcal{E} S \mathcal{E} S^{\cdot} L$

$\mathrm{CDCl}_{3}$ 


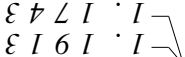
$z \varepsilon<t \cdot I-$ $606 \mathrm{t} \cdot I-$ $280 \mathrm{~S}^{\circ} \mathrm{IL}$

$69 \angle 9{ }^{\circ} z$ $0+69 \cdot z$ O I I L L $Z$ $+8 z<\cdot z$ $+s+L$

S $29 L \cdot z$ $26 \angle L \cdot 2$
$009 Z^{\circ} L$ $\rightarrow S \mathcal{E} \mathcal{E} \cdot L-=$ $O S \subseteq \mathcal{E} \cdot L-\equiv$ $8 Z \angle t^{\circ} \angle-$ $026 t^{\circ} L$ EI IS $L$

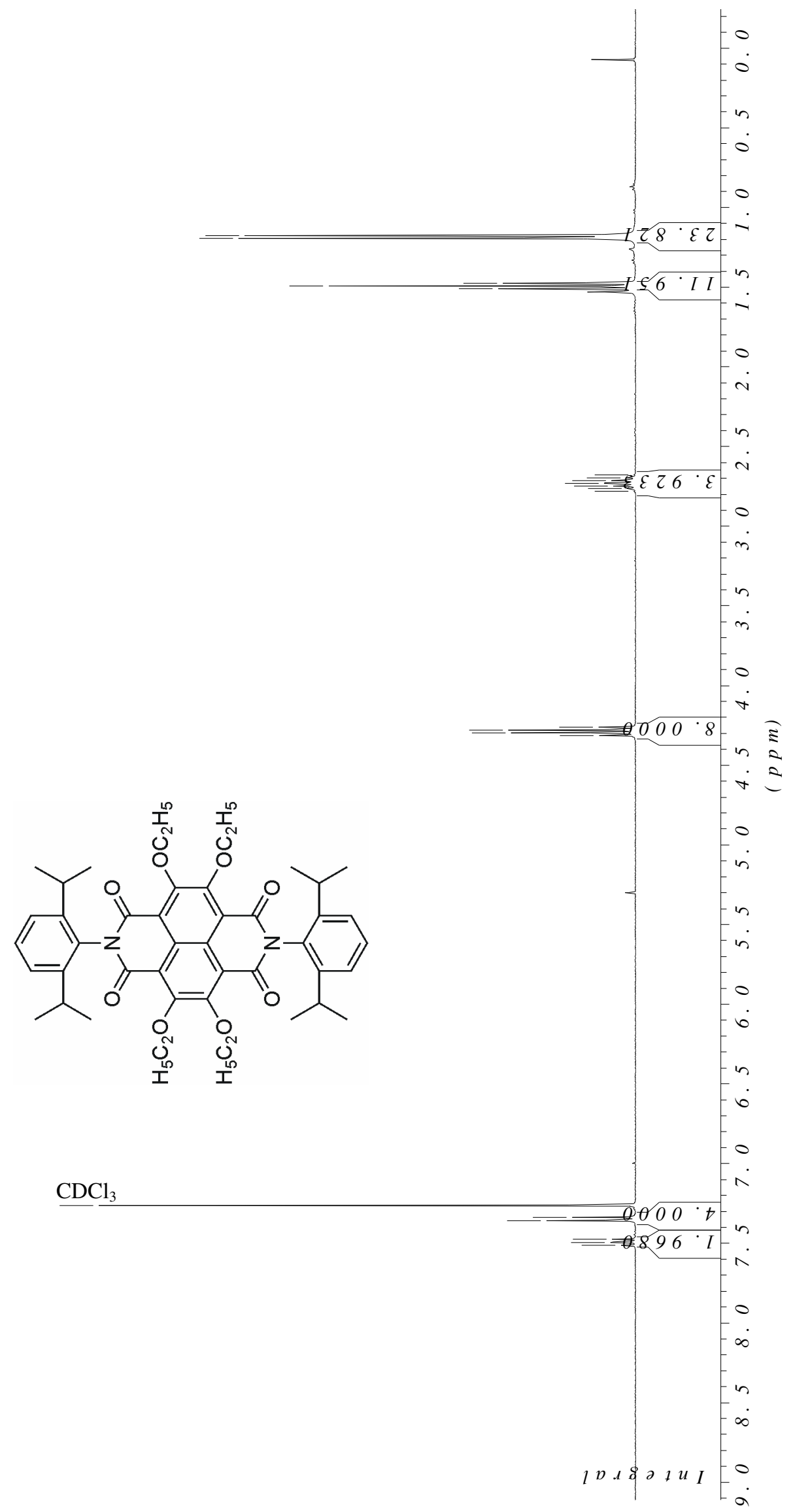


NDI 7

$I \mathcal{E} 8 I^{\circ}$

$\begin{array}{llll}1 & 0 & 0 & \mathrm{Z}\end{array}$

$\begin{array}{lllll}6 & I & 2 & Z\end{array} \cdot 1-$

$\begin{array}{llll}S & 0 & t & Z \\ L & S & S & I\end{array}$

$\varepsilon z \leq 9^{\circ} z-$

$\varepsilon 0<9 \cdot z$

$9 \angle 89$

$\angle D O \angle$

$0 z z L$

I $6 \varepsilon<\cdot z$

$6+S \angle \cdot z-$

$80<6 \cdot 2-$

$+686^{\circ} \mathrm{z}-$

\begin{tabular}{lllll}
$I$ & 8 & 0 & 0 & $\cdot$ \\
$\angle$ & 9 & 2 & 0 & $\varepsilon$ \\
\hline
\end{tabular}

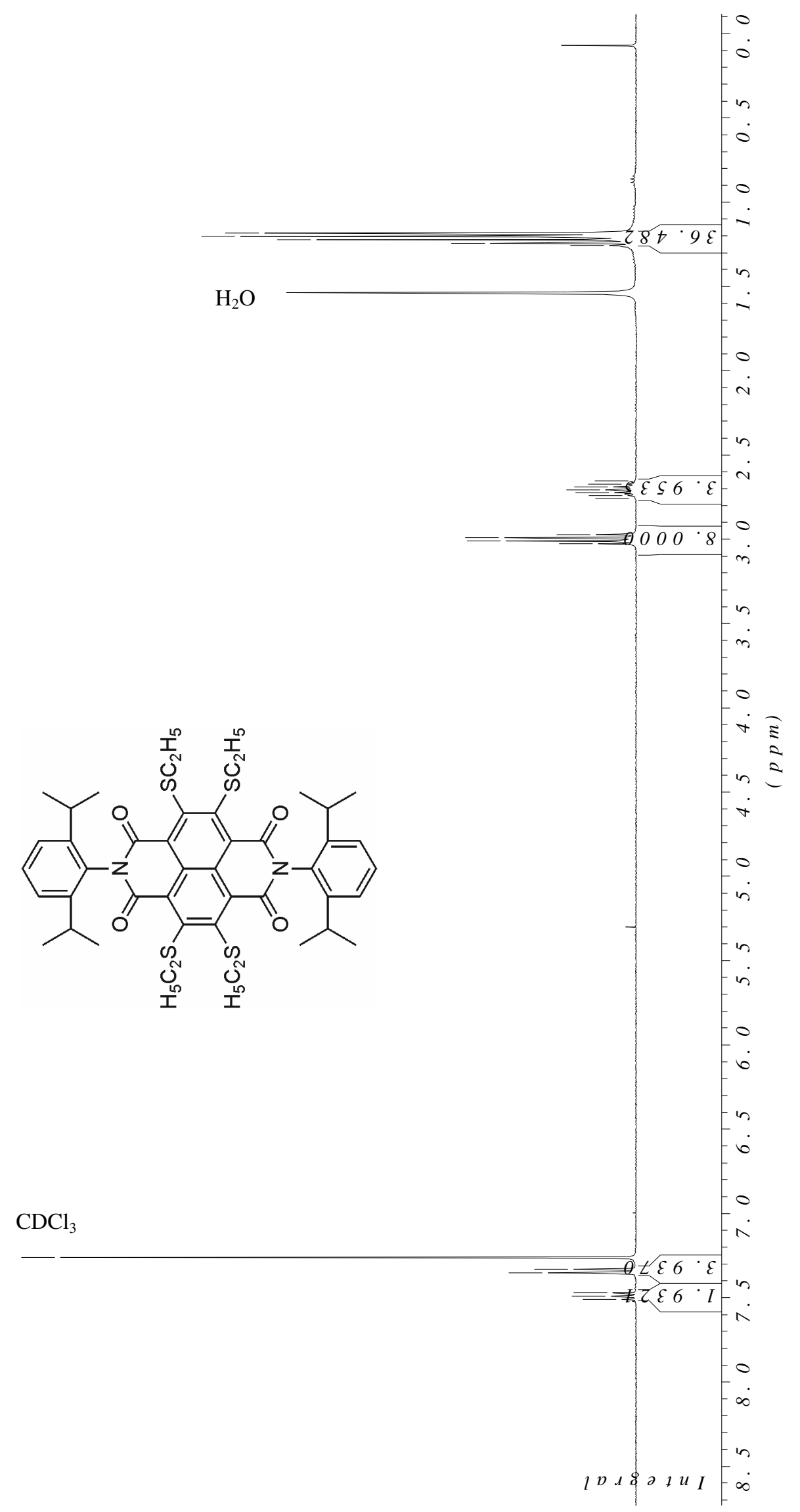

$0092 \cdot \angle-$

$\angle O E \mathcal{E} \cdot \angle-$

$00 S \mathcal{E}: L-F$

$\varepsilon 69 \mathrm{t}^{\circ} \mathrm{L}-$

$\begin{array}{lllll}9 & 8 & 8 & t \\ I & 8 & 0 & 5 & L\end{array}$ 
NDI 8

$\left.\begin{array}{lllll}t & v & 0 & L & u \\ t & I & 0 & 8 & 0\end{array}\right]$

$58 I 8 \cdot 0$

$\angle Z \angle I I^{\circ} I-$

$\begin{array}{llll}\angle & 6 & 8 & I \\ \angle & I & E & Z\end{array} \cdot 1$

$+\angle \mathcal{E} Z{ }^{\circ} I-$

$s 9+2 \cdot I-\equiv$

S $85 Z \cdot I-$

$8 I \angle Z \cdot I-$

$29<2 \cdot I-E$
$S 6 t t \cdot I-E$

$+69 t^{\prime} \cdot I-$

S98 $\mathrm{t}^{\circ} \mathrm{I}-\mathrm{F}$

$t S O S S^{\circ} \cdot I-$

$[Z Z S \cdot I]$

$60+9 \cdot 2$

$08559 \cdot 2-$

I $2699^{\circ} 2$

$+60<\cdot$

$592<\cdot z-$

$9 \angle t<\cdot z$

S90t' $\mathcal{E}-$

$8+2 t \cdot \mathcal{E}-$

$8 z+t \cdot \mathcal{E}$

$0092 \cdot \angle$

$6 \angle S \mathcal{E} \cdot \angle-F$

$+\angle \angle \mathcal{E} \cdot \angle-F$

$9 \angle 8 t^{\circ} \angle-$

$\begin{array}{lllll}2 & 9 & 0 & S & \cdot L \\ I & 9 & 2 & S & L\end{array}$

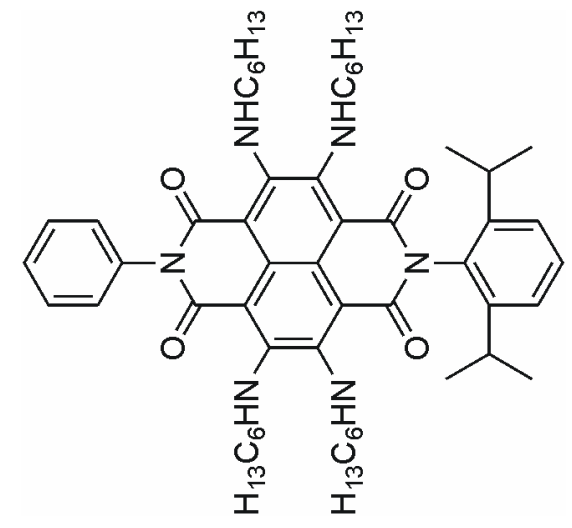

$ミ$
2
2

in

0

6

in.

0

$\mathrm{CDCl}_{3}$

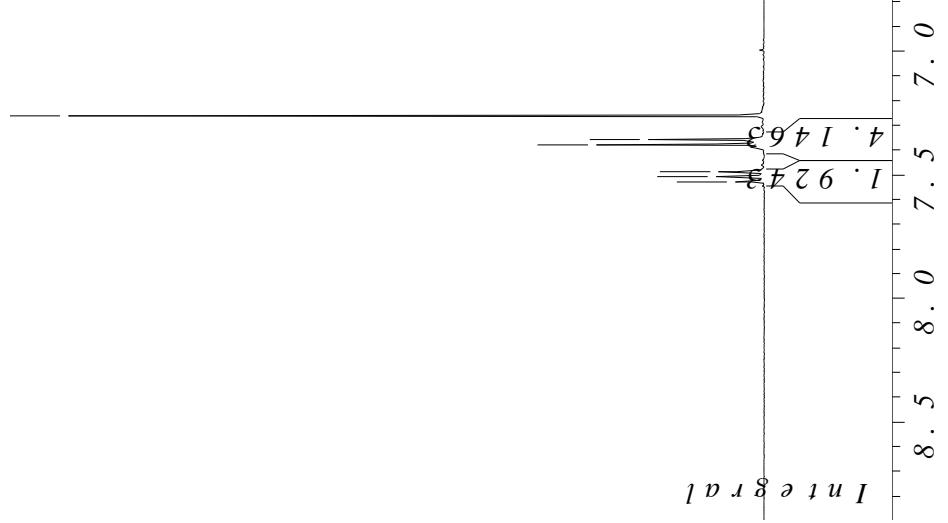


NDI 9

$2+9 I \cdot I-$

$608 I \cdot I L$

TMS

$88 \angle 9^{\circ} \mathrm{Z}$

$s \varsigma 69^{\circ} z$

9 ZI L $z_{-}$

$962 \angle \cdot 2$
$\angle 9+\angle \cdot z$

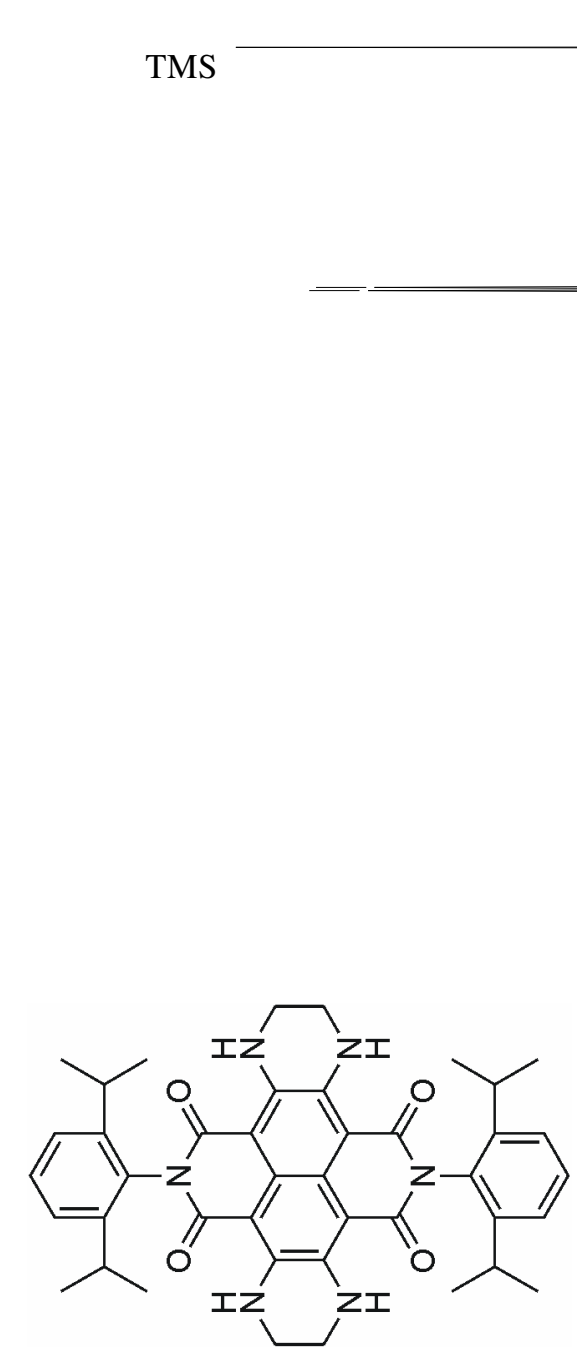

$I S+9$
$8 S S 9$

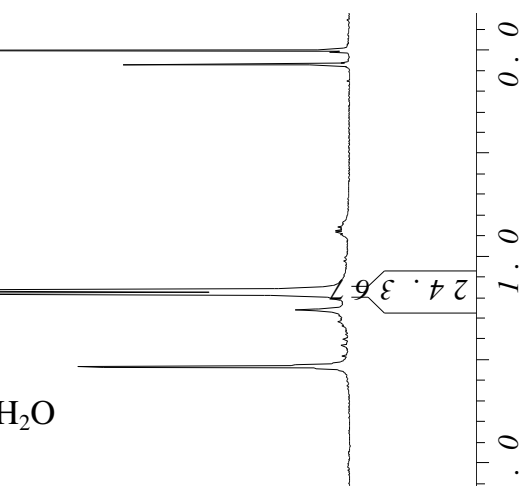

0

i

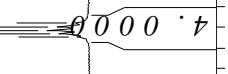

$\mathrm{CDCl}_{3}$

$\begin{array}{llll}0 & 0 & 9 & Z^{\prime} \\ \tau & + & \mathcal{E} & \mathcal{E}\end{array}$

$+\mathcal{E} S \mathcal{E} \cdot L-$

$+9 S t^{\circ} \angle-$

$\angle t \angle t \cdot \angle-$

$99 \angle t \cdot \angle-$

$6+6 t \cdot L-$

I $828 \cdot 6$

.

$1828 \cdot 6$

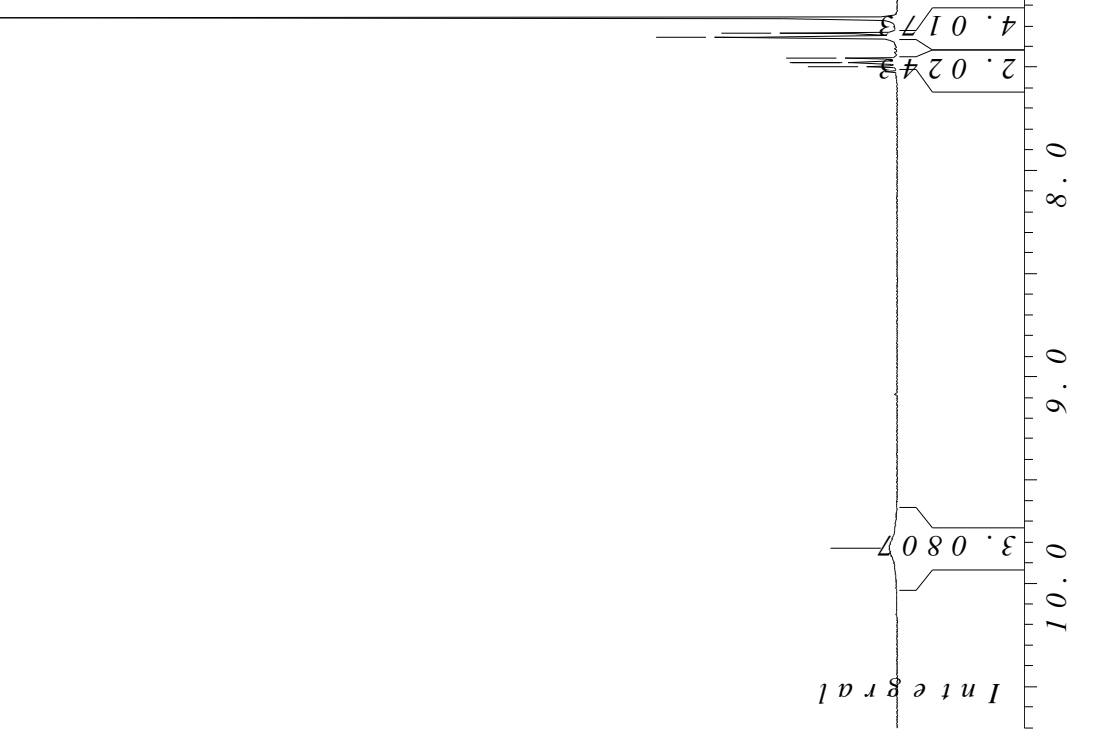

